Check for updates

Cite this: Chem. Sci., 2019, 10, 7251

๑ All publication charges for this article have been paid for by the Royal Society of Chemistry

\section{Substituent-controlled, mild oxidative fluorination of iodoarenes: synthesis and structural study of aryl I(III)- and I(v)-fluorides $\uparrow$}

\author{
Joel Häfliger, $\$$ Cody Ross Pitts, D \& Dustin Bornemann, Roland Käser, \\ Nico Santschi, (D) Julie Charpentier, Elisabeth Otth, Nils Trapp, René Verel, \\ Hans Peter Lüthi and Antonio Togni (D) *
}

\begin{abstract}
We report a mild approach to the synthesis of difluoro(aryl)- $\lambda^{3}$-iodanes (aryl-IF compounds) and tetrafluoro(aryl) $-\lambda^{5}$-iodanes (aryl- $\mathrm{IF}_{4}$ compounds) using trichloroisocyanuric acid (TCICA) and potassium fluoride (KF). Under these reaction conditions, selective access to either the I(III)- or I(V)-derivatives is predictable based solely on the substitution pattern of the iodoarene starting material. Moreover, the discovery of this TCICA/KF approach prompted detailed dynamic NMR, kinetic, computational, and crystallographic studies on the relationship between the $\mathrm{IF}_{2}$ group and the ortho-substituents on carefully designed probe molecules. It was during these experiments that the role of the orthosubstituent in inhibiting further oxidative fluorination of I(III)-compounds to I(V)-compounds during the reaction with TCICA and KF was revealed. Additionally, a notable exception to this empirical trend is

discussed herein.
\end{abstract}

Received 3rd May 2019

Accepted 23rd May 2019

DOI: $10.1039 / \mathrm{c} 9 \mathrm{sc} 02162 \mathrm{k}$

rsc.li/chemical-science
Oxidative fluorination chemistry has often stipulated the use of harsh reagents. Regarding the fluorination of aryl iodides, synthetic limitations arguably have had significant downstream effects on the ability to study structure and reactivity of difluoro(aryl)- $\lambda^{3}$-iodanes (aryl- $\mathrm{IF}_{2}$ compounds) and, to an even greater extent, tetrafluoro(aryl)- $\lambda^{5}$-iodanes (aryl- $\mathrm{IF}_{4}$ compounds) in the last several decades. ${ }^{1}$ Aside from two important exceptions from the Shreeve ${ }^{2}$ and Gilmour ${ }^{3}$ laboratories (employing Selectfluor in the synthesis of electron-rich ${\text { aryl- } \mathrm{IF}_{2}}_{2}$ compounds), the vast majority of methods for aryl- $\mathrm{IF}_{2}$ synthesis rely on $\mathrm{F}_{2}$, a source of $\mathrm{HF}$, or a number of other notoriously hazardous reagents (e.g. $\mathrm{SF}_{4}, \mathrm{XeF}_{2}$, etc.). ${ }^{1,4,5}$ Aryl- $-\mathrm{IF}_{4}$ synthesis is equally if not more challenging. ${ }^{1,6}$

As one possible solution to this accessibility problem, we present a mild, safe, and inexpensive oxidative fluorination of aryl iodides that requires only trichloroisocyanuric acid (TCICA) and potassium fluoride. Somewhat serendipitously, we found that the selectivity between aryl- $\mathrm{IF}_{2}$ and aryl- $\mathrm{IF}_{4}$ formation can be controlled reliably by the substitution pattern of the arene (Fig. 1). This discovery was made during an in-depth study on the relationship between the $\mathrm{IF}_{2}$ group and ortho-substituents,

Department of Chemistry and Applied Biosciences, ETH Zürich, Vladimir-Prelog-Weg 2, 8093 Zürich, Switzerland. E-mail: atogni@ethz.ch

$\dagger$ Electronic supplementary information (ESI) available. CCDC 1913138-1913140. For ESI and crystallographic data in CIF or other electronic format see DOI: $10.1039 / \mathrm{c} 9 \mathrm{sc} 02162 \mathrm{k}$

$\ddagger$ These authors contributed equally. whereby we conducted several variable-temperature NMR, computational, and crystallographic analyses, reported herein.

\section{Reaction design and optimization}

Originally, the reaction design was inspired by our recent foray into aryl- $\mathrm{SF}_{4} \mathrm{Cl}$ compound synthesis, whereby we accomplished a mild, gas reagent-free oxidative fluorination of diaryl disulfides using TCICA, KF, and catalytic TFA. ${ }^{7}$ Using a similar approach, we began screening with iodobenzotrifluoride isomers: this would potentially allow analysis of the material balance of the reaction mixtures by ${ }^{19} \mathrm{~F}$ NMR. After stirring the reaction mixtures at $\mathrm{rt}$ in MeCN for $24 \mathrm{~h}$ using TCICA (1.0 equiv.), KF (3.0 equiv.), and TFA ( $10 \mathrm{~mol} \%)$, we made two important observations: (1) when making the NMR samples in the fume hood (i.e. not under rigorously air- and moisture-free conditions), we noticed rapid formation of a precipitate in the

substitution-controlled access to fluorinated I(III) and I(V) species

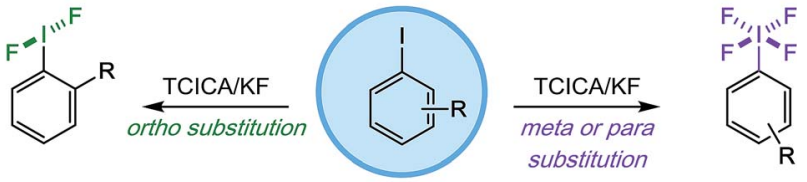

synthesis and evaluation of ortho-substituent effect on aryl-IF ${ }_{2}$ compounds

Fig. 1 Mild and predictable access to aryl-IF $F_{2}$ and aryl-IF 4 compounds based on iodoarene substitution pattern. 
filtered samples that employed the meta- and para-iodobenzotrifluoride isomers as substrates, but not in the ortho-isomer sample (specifically upon addition of an internal standard solution with slightly wet $\mathrm{CD}_{3} \mathrm{CN}$ ) and (2) although ${ }^{19} \mathrm{~F}$ NMR analysis indeed showed formation of the corresponding difluoro(aryl)- $\lambda^{3}$-iodanes (i.e. aryl- $\mathrm{IF}_{2}$ compounds) in all cases, the products were observed in only trace yields for the meta-and para-isomers and in about $20 \%$ for the ortho-isomer, the majority of the observable material balance being unreacted starting material.

At the time, we assumed the identity of the precipitate was the corresponding iodosoarene for each the meta- and paraiodobenzotrifluoride isomers. In this light, our observations suggested that the ortho-substituted aryl- $\mathrm{IF}_{2}$ compound may be less susceptible to degradation, and thus easier to handle during the screening process. Accordingly, we continued to screen for optimized conditions using ortho-iodobenzotrifluoride 1 (Table 1) (notably, it was not until much later in the study that we determined the precipitate was not primarily a byproduct of aryl- $\mathrm{IF}_{2}$ compound hydrolysis, but rather a byproduct of aryl- $\mathrm{IF}_{4}$ compound hydrolysis, as discussed in more detail below. While our initial assumption was erroneous, the following series of experiments led us to this discovery of the effect of substitution on oxidative fluorination).

Immediately, we discovered that although an acid catalyst was beneficial for aryl- $\mathrm{SF}_{4} \mathrm{Cl}$ formation in our previous work, it had no positive impact on aryl- $\mathrm{IF}_{2}(2)$ product formation. Yet, we found that using TCICA and KF in slight excess had a considerable impact, bringing the yield up to $79 \%$ at room temperature in the absence of a catalyst. Next, we attempted the reaction at slightly elevated temperatures. To our surprise, the aryl- $\mathrm{IF}_{2}$ product 2 not only survived heating the reaction mixture to $40{ }^{\circ} \mathrm{C}$ in a borosilicate vial for $24 \mathrm{~h}$, but it formed in $94 \%$ yield by ${ }^{19}$ F NMR (using 4.0 equiv. TCICA and 6.0 equiv. KF).

Table 1 Screening for optimal reaction conditions ${ }^{a}$

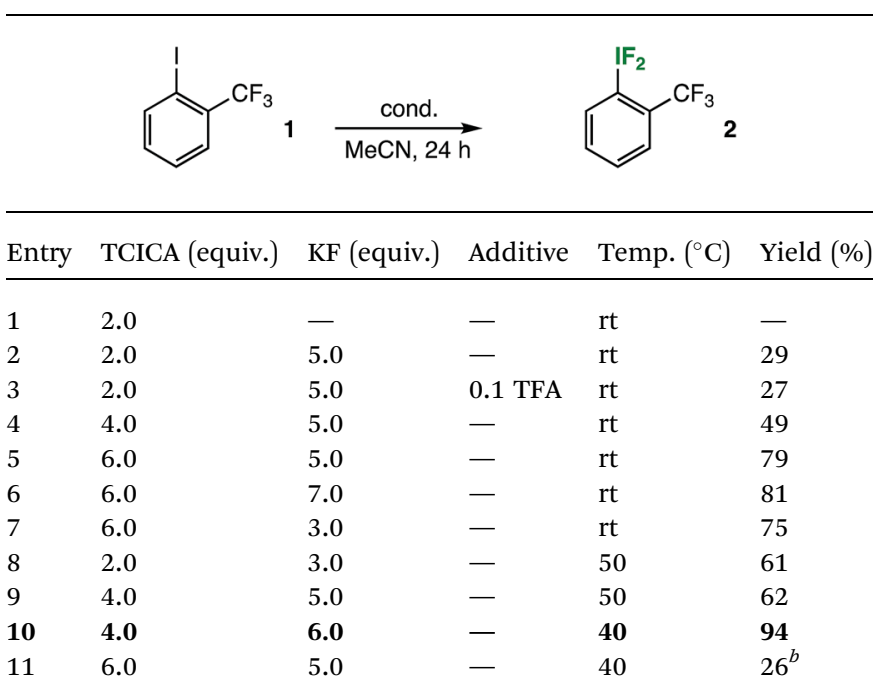

${ }^{a}$ Yields determined by ${ }^{19} \mathrm{~F}$ NMR using fluorobenzene as an internal standard. ${ }^{b}$ MeCN not dry.
We proceeded to screen with these optimized conditions. Additionally, we found that: (1) the reaction does not proceed in solvents such as DMF, DCM, EtOAc, and toluene, and the product yield is poor when the MeCN is not dry; (2) N-chlorosuccinimide and $N$-chlorophthalimide are not suitable replacements for TCICA under optimized conditions (i.e. no aryl- $\mathrm{IF}_{2}$ product formation was observed in either case); (3) reaction times of less than $24 \mathrm{~h}($ e.g. $16 \mathrm{~h})$ may result in inferior product yields; and (4) the aryl- $\mathrm{IF}_{2}$ product, over 2-3 days, decomposes more quickly when exposed to light in a filtered solution of the crude reaction mixture in $\mathrm{CD}_{3} \mathrm{CN}$ at room temperature.

\section{Substrate scope of difluoro(aryl)- $\lambda^{3}-$ iodanes and the importance of ortho- substitution}

The most remarkable aspect of the optimization process was the consistent absence of the expected decomposition product 2-iodosobenzotrifluoride - as determined by ${ }^{19} \mathrm{~F}$ NMR and lack of precipitate formation in the NMR tube. Suspecting that the substitution pattern plays an important role, we investigated the substrate scope by first holding the ortho-trifluoromethyl substituent constant (Table 2).

In doing so, we noted that the reaction tolerates substrates adorned with additional electron-withdrawing substituents, such as fluorine, chlorine, bromine, esters, and nitro groups (compounds 3-7). Also, ortho-disubstitution does not inhibit aryl- $\mathrm{IF}_{2}$ formation, as demonstrated by products 8 and $\mathbf{9}$. This substrate class has been popularized recently as a privileged platform to access chiral aryl- $\mathrm{IF}_{2}$ reagents. ${ }^{8}$ On the other hand, the reaction does not tolerate strong electron-donating groups, as these substrates are prone to competitive ring and/or benzylic chlorination in the presence of TCICA. ${ }^{9}$ Also, aryl- $\mathrm{IF}_{2}$ product formation is not observed (or observed in very poor yield) in the presence of substituents with acidic protons, such as carboxylic acids or secondary amides.

Beyond ortho-trifluoromethyl substitution, we also discovered that aryl- $\mathrm{IF}_{2}$ compounds containing other fluorinated substituents, such as ortho-trifluoromethoxy substituents (10) or ortho-difluoro substituents (11 and 12), may be synthesized in good yields under our conditions (Table 2).

Subsequently, we continued to vary systematically the orthosubstituent to electron-withdrawing groups of various sizes, such as halogens (compounds 13-15), an ester (compound 16), and a nitro group (compound 17). The reaction performed well in all cases. Additionally, we found that mild donating groups (e.g. a methyl group) in the ortho-position are compatible under reaction conditions. That is, no notable background benzylic or ring chlorination was observed in the synthesis of compound 18.

In nearly all cases, no decomposition or precipitation was observed upon analyzing the aryl- $\mathrm{IF}_{2}$ products in Table 2 , which is in stark contrast to the aforementioned meta- and parasubstituted substrates. This prompted an important question: 
Table 2 Substrate scope of ortho-substituted aryl-IF 2 compounds. Yields were determined by ${ }^{19} \mathrm{~F}$ NMR using either fluorobenzene or benzotrifluoride as an internal standard and verified by analysis of the material balance

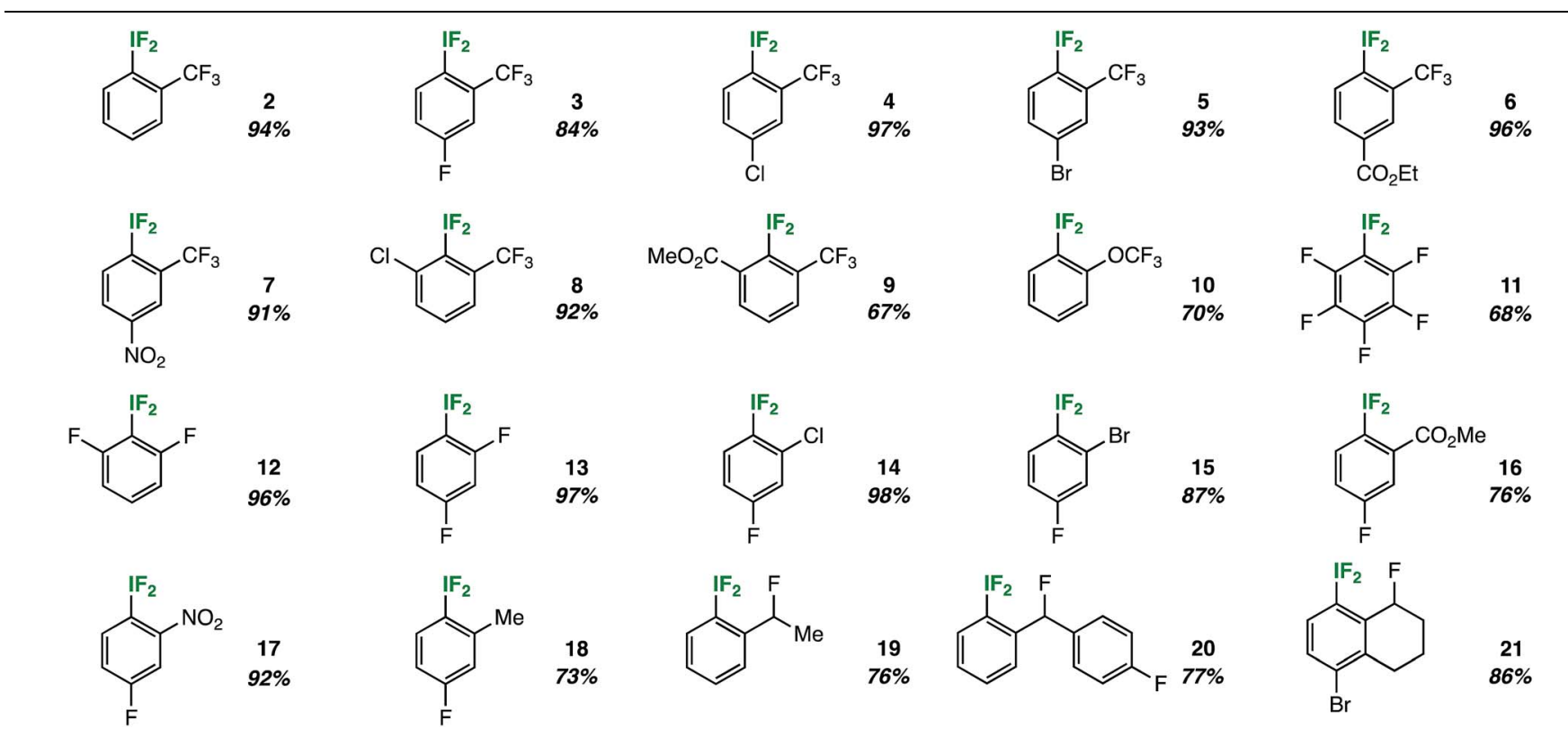

is ortho-substitution, in general, somehow important in relation to the hydrolytic stability of aryl- $\mathrm{IF}_{2}$ compounds?

\section{Probing an $\mathrm{IF}_{2}$ interaction}

Preliminary evidence for an interaction between the $\mathrm{IF}_{2}$ group and the ortho-substituent stems from the observed $J$-coupling of $3.2 \mathrm{~Hz}$ between the $\mathrm{IF}_{2}$ fluorine atoms and $\mathrm{CF}_{3}$ fluorine atoms in the ${ }^{19} \mathrm{~F}\left\{{ }^{1} \mathrm{H}\right\}$ NMR spectrum of compound 2 . Note that we also observed a $J$-coupling of $2.3 \mathrm{~Hz}$ between the $\mathrm{IF}_{2}$ fluorine atoms and the ortho-fluorine atoms in compound 12. Although initial attempts to clarify the results through ${ }^{19} \mathrm{~F}^{19}{ }^{19} \mathrm{~F}$ NOESY experiments were unsuccessful, these $J$-values are likely attributed to a lone-pair interaction.

We examined the relationship between the $\mathrm{IF}_{2}$ and $\mathrm{CF}_{3}$ group in compound 2 further using DFT calculations. Geometry optimizations were performed using Gaussian ${ }^{10}$ at the $\mathrm{wB} 97 \mathrm{xD} /$ cc-pvdz level of theory, ${ }^{11}$ using a cc-pvdz-PP basis set for the iodine atom, ${ }^{12}$ on the ortho-, meta-, and para-trifluoromethyl aryl- $-\mathrm{IF}_{2}$ isomers $\mathbf{2 , 2 2}$, and $\mathbf{2 3}$. Interestingly, compound $\mathbf{2}$ is predicted to be the least thermodynamically stable by $9.8 \mathrm{kcal} \mathrm{mol}^{-1}$ (for 22 and $23, \Delta E \approx 0$ ), which rules out the possibility of a stabilizing interaction between the fluorine atoms on the $\mathrm{CF}_{3}$ group and the iodine atom. A closer look at the structures reveals similar bond distances, angles, and natural charges predicted for all isomers, but one property is drastically different - the $\mathrm{C}_{\text {ortho }}-\mathrm{C}_{\text {ipso }}-\mathrm{I}-\mathrm{F}$ dihedral angle (Table 3). In compounds 22 and $\mathbf{2 3}$, the lowest energy conformations both have dihedral angles of virtually $0^{\circ}$, while the dihedral angle of compound 2 is $40^{\circ}$ - forced out of plane with the ring. In this particular instance, the preferred angle of $0^{\circ}$ in 22 and 23 calculated in the gas phase is likely attributed to a favorable interaction between the iodine lone pairs and the $\pi$-system, which the ortho-substituent in $\mathbf{2}$ seems to hinder. The calculated angles notably differ from the preferred orientation of the $\mathrm{IF}_{2}$ group (and many related $\mathrm{IX}_{2}$ groups) in a number of X-ray crystal structures.

Overall, this provides little indication of what is going on in solution, but suggests that the $\mathrm{CF}_{3}$ group may, in fact, have an influence on the preferred orientation of the $\mathrm{IF}_{2}$ group. Specifically, in solution, this could affect the rotation about the C-I bond. One postulate could be that if the preferred $\mathrm{IF}_{2}$ orientation is out of the plane of the ring, this would leave the iodine atom less susceptible to nucleophilic attack, thus offering one explanation for our observations thus far. An analogy may be drawn to the stabilizing effects of the ortho-methyl groups on the $\mathrm{SF}_{3}$ moiety in Fluolead, for instance. ${ }^{13}$

\section{Dynamic NMR probe}

In order to examine the influence of the ortho-substituent on the conformation of the $\mathrm{IF}_{2}$ group in solution, we designed probe molecules suitable for variable-temperature NMR (VT-NMR) studies. Initially, we synthesized compounds $19^{14}$ and $20^{14 a, 15}$ (Table 2), each with a stereogenic center and fluorine atom in the benzylic position, as possible probes. Theoretically, if the $\mathrm{C}-\mathrm{I}$ bond in these molecules is not freely rotating, the fluorine atoms on the $\mathrm{IF}_{2}$ moiety would become diastereotopic, thus leading to two spectroscopically distinct ${ }^{19} \mathrm{~F}$ NMR signals.

In MeCN, no dynamic behavior was observed in the $\mathrm{IF}_{2}$ signal by ${ }^{19} \mathrm{~F}$ NMR down to approximately $233 \mathrm{~K}$ for compound 19. However, this result may be complicated by free rotation about the $\mathrm{C}_{\text {ortho }}-\mathrm{C}_{\text {benzylic }}$ bond. In an attempt to limit this rotation, we replaced the methyl group in the benzylic position with a larger, aryl substituent (compound 20); still, no dynamic behavior was observed down to $233 \mathrm{~K}$. 
Table 3 Comparing properties of isomers 2, 22, and 23 via DFT calculations ${ }^{a}$

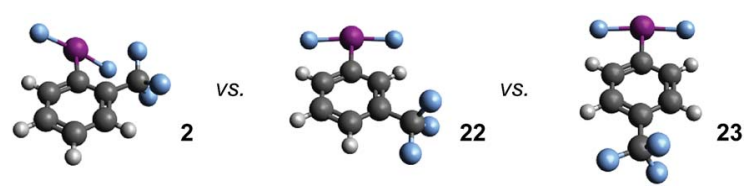

\begin{tabular}{|c|c|c|c|c|c|c|c|c|}
\hline Subs. & $d(\mathrm{C}-\mathrm{I})$ & $d(\mathrm{I}-\mathrm{F})$ & $q_{\mathrm{C}}$ & $q_{\mathrm{I}}$ & $q_{\mathrm{F}}$ & $\theta_{\mathrm{C}-\mathrm{I}-\mathrm{F}}$ & $\theta_{\mathrm{F}-\mathrm{I}-\mathrm{F}}$ & $\phi_{\mathrm{C}-\mathrm{C}-\mathrm{I}-\mathrm{F}}{ }^{b}$ \\
\hline$o-\mathrm{CF}_{3}$ & $2.13 \AA$ & $2.01 \AA^{c}$ & -0.248 & 1.419 & $-0.627^{d}$ & $86^{\circ e}$ & $172^{\circ}$ & $40^{\circ f}$ \\
\hline$m-\mathrm{CF}_{3}$ & $2.13 \AA$ & $2.02 \AA^{g}$ & -0.251 & 1.409 & -0.628 & $87^{\circ}$ & $174^{\circ}$ & $0^{\circ}$ \\
\hline
\end{tabular}

${ }^{a}$ Calculations peformed at $\mathrm{wB} 97 \mathrm{xD} / \mathrm{cc}-\mathrm{pvdz}$, with a cc-pvdz-PP basis set used for the iodine atom. $\mathrm{C}$ refers to ipso carbon atom and $\mathrm{F}$ refers to fluorine atom bound to iodine, unless otherwise specified. ${ }^{b}$ Dihedral angle of $\mathrm{C}_{\text {ortho }}-\mathrm{C}_{\mathrm{ipso}}-\mathrm{I}-\mathrm{F} .{ }^{c}$ Average between 2.00 and 2.02 (not equal bond lengths). ${ }^{d}$ Average between -0.621 and $-0.633 .{ }^{e}$ Average between $85^{\circ}$ and $87^{\circ}{ }^{f}{ }^{f} \mathrm{~F}$ atom proximal to substituent (dihedral angle is $38^{\circ}$ for distal F). ${ }^{g}$ Structure is nearly symmetric about F-I-F (mirror plane). ${ }^{h}$ Structure is symmetric about F-I-F (mirror plane).

Assuming rotation about the $\mathrm{C}_{\text {ortho }}-\mathrm{C}_{\text {benzylic }}$ bond was, indeed, still complicating the picture, we synthesized a lesstrivial tetrahydronaphthalene derivative $\mathbf{2 1}$ (Table 2) in order to eliminate this variable by locking the benzylic position into place with a more rigid ring structure (Fig. 2, top panel). This probe molecule 21 was made in 8 steps in $9 \%$ overall yield from commercially available 5,6,7,8-tetrahydro-1-naphthylamine $\mathbf{2 4}$ (see ESI $\dagger$ for details). ${ }^{\mathbf{1 4}, 16}$ Not only was it designed to inhibit $\mathrm{C}_{\text {ortho }}-\mathrm{C}_{\text {benzylic }}$ bond rotation, but $\mathbf{2 1}$ was also adorned with an electron withdrawing group (i.e. a bromine atom) in the 4position to deactivate the aromatic ring and benzylic position distal to the $\mathrm{IF}_{2}$ moiety toward background chlorination from TCICA (Fig. 2, bottom panel).

To our satisfaction, we observed significant line broadening of the $\mathrm{IF}_{2}$ signal in 21 upon cooling the sample and approached the coalescence temperature, which was likely just below $233 \mathrm{~K}$. As this is near the freezing point of the solvent, we concentrated the reaction mixture and redissolved the residue in deuterated dichloromethane to access lower temperatures.
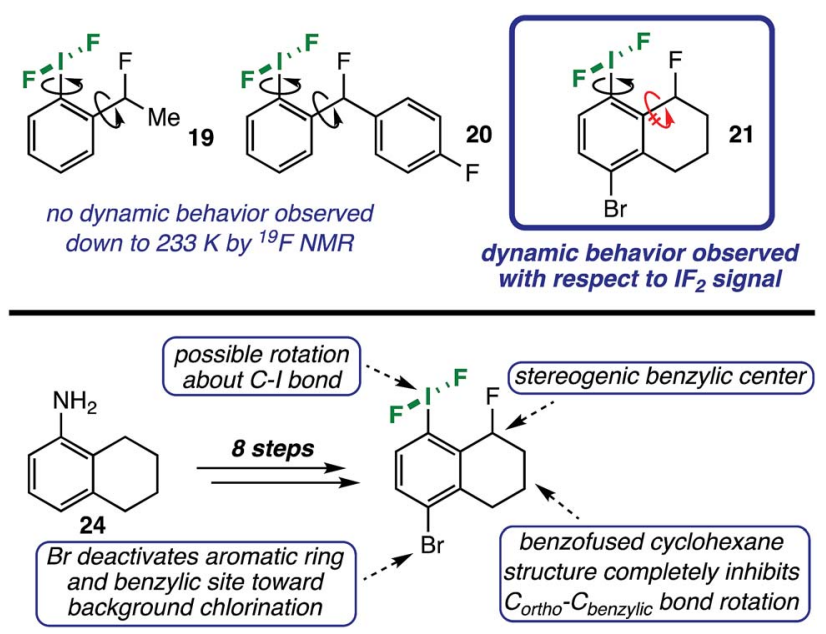

Fig. 2 (Top panel) Probe molecules used to investigate rotation of $\mathrm{IF}_{2}$ moiety about $\mathrm{C}-\mathrm{I}$ bond via VT-NMR. (Bottom panel) Highlighting design features of probe molecule 21 .
Upon cooling a solution of 21 in $\mathrm{CD}_{2} \mathrm{Cl}_{2}$, we determined a coalescence temperature $\left(T_{\mathrm{C}}\right)$ of $228 \mathrm{~K}$ and observed complete separation of the " $\mathrm{IF}_{2} "{ }^{19} \mathrm{~F}$ NMR signal into two distinct doublets with a clear roofing effect at temperatures below $202 \mathrm{~K}$ (Fig. 3). ${ }^{17}$ Here, the fluorine atoms bound to iodine are seen as diastereotopic on the NMR time-scale with ${ }^{2} J_{\mathrm{FF}}=98.3 \mathrm{~Hz}$, and the distance between the two signals, i.e. $\left|\left(\nu_{\mathrm{A}}-\nu_{\mathrm{B}}\right)\right|$, is $1152 \mathrm{~Hz}$. Note that no coupling constant between the benzylic fluorine atom and either of the iodine-bound fluorine atoms is observable at any temperature within our data set; however, as this coupling constant is expected to be small ( $c a .2-3 \mathrm{~Hz}$ ), it would likely be subsumed in the broader parent signals.

Additionally, it is important to state that we did not observe dynamic activity of either the benzylic fluorine or hydrogen atoms by either ${ }^{19} \mathrm{~F}$ or ${ }^{1} \mathrm{H}$ NMR at any temperature down to 168 $\mathrm{K}$, indicating that the benzo-fused cyclohexane ring-flipping dynamics have a negligible impact on the $\mathrm{IF}_{2}$ dynamics.

At temperatures below $202 \mathrm{~K}$, we also noted an apparent convergence of the two $\mathrm{IF}_{2}{ }^{19} \mathrm{~F}$ signals down to $168 \mathrm{~K}$, with $\mid\left(\nu_{\mathrm{A}}-\right.$ $\left.\nu_{\mathrm{B}}\right) \mid=989 \mathrm{~Hz}$. This is likely due to a more dramatic temperaturedependent shift of one of the fluorine signals over the other. ${ }^{\mathbf{1 8}}$ Although this phenomenon is not surprising, it complicates the accurate determination of rate constants $(k)$, and thus activation energy $\left(E_{\mathrm{a}}\right)$, as the equations for the $T=T_{\mathrm{C}}$ and $T>T_{\mathrm{C}}$ temperature regimes both depend on the maximum chemical shift difference $\left|\left(\nu_{\mathrm{A}}-\nu_{\mathrm{B}}\right)\right|$ (see ESI $\dagger$ for details). ${ }^{19}$ Accordingly, we have analyzed our data using two different assumptions: (1) $\left|\left(\nu_{\mathrm{A}}-\nu_{\mathrm{B}}\right)\right|=$ signal separation we observed at lowest measurable temperature $(989 \mathrm{~Hz})$ and $(2)\left|\left(\nu_{\mathrm{A}}-\nu_{\mathrm{B}}\right)\right|=$ largest signal separation we observed $(1152 \mathrm{~Hz})$ in our data set. Under these assumptions, we have determined activation energies from Arrhenius analyses, respectively, of $7.0 \pm 0.9$ and $7.4 \pm$ $0.9 \mathrm{kcal} \mathrm{mol}^{-1}$. We also note that respective enthalpies of activation $\left(\Delta H^{\ddagger}\right)$ of $6.5 \pm 0.9$ and $6.9 \pm 0.9 \mathrm{kcal} \mathrm{mol}^{-1}$ and entropies of activation $\left(\Delta S^{\ddagger}\right)$ of $-13 \pm 4$ and $-11 \pm 4 \mathrm{cal} \mathrm{mol}^{-1} \mathrm{~K}^{-1}$ were calculated for each assumption via Eyring plots. Considering there is a convergence of the $\Delta\left|\left(\nu_{\mathrm{A}}-\nu_{\mathrm{B}}\right)\right|$ that is exacerbated at lower temperatures, we believe that assumption (2) may be 


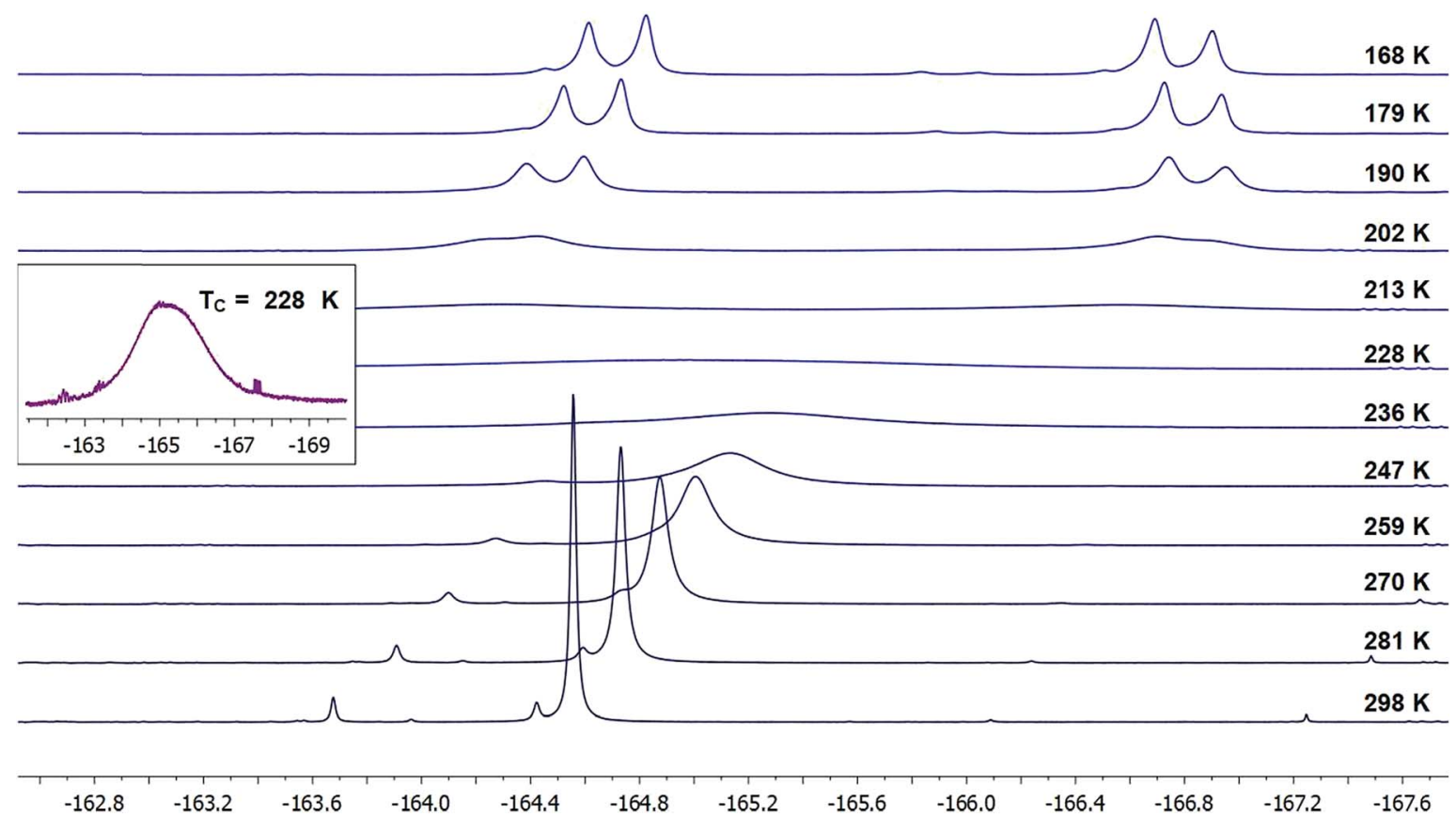

Fig. 3 Stacked ${ }^{19} \mathrm{~F}$ NMR spectra of the $\mathrm{IF}_{2}$ signal in probe molecule 21 over a range of temperatures from $298 \mathrm{~K}$ to $168 \mathrm{~K}$. A zoomed-in spectrum is displayed for the coalescence temperature $\left(T_{C}\right)$ at $228 \mathrm{~K}$.

more appropriate, though both assumptions lead to values that are very close in agreement.

Although these thermodynamic parameters presumably describe rotation about the C-I bond, one must consider alternative mechanisms. For one, we ruled out the possibility of an intermolecular exchange mechanism by performing the VTNMR studies at different concentrations (see ESI $\uparrow$ for details). ${ }^{20}$ A more challenging issue to address, however, is pseudorotation. Very few detailed dynamic NMR studies on I(III) compounds (and, notably, none on aryl- $\mathrm{IF}_{2}$ compounds) have surfaced in the literature, but one can attribute the observed dynamic activity of diacetoxyaryl- and triaryl-substituted iodanes to pseudorotation in the works of Reich and Cooperman $^{21}$ and Ochiai and coworkers, ${ }^{20}$ respectively. Their probe molecules are incomparable to compound 21 in terms of ligand substitution patterns, core skeletons, etc.; thus, there can be no straightforward comparison of our experimentally-determined thermodynamic values to the existing literature. In order to probe the likelihood of C-I rotation being the relevant mechanism over pseudorotation for our aryl-IF ${ }_{2}$ model compound 21, we turned to DFT calculations.

\section{DFT analysis of rotational barrier}

Compound 21 was optimized at the wB97xD/cc-pvdz level of theory (cc-pvdz-PP for the iodine atom), and then the $\mathrm{IF}_{2}$ group was rotated $180^{\circ}$ about the $\mathrm{C}-\mathrm{I}$ bond in $12^{\circ}$ increments. The structure was relaxed at each step of the rotation scan to determine the lowest energy conformations. From the plotted potential curve, we calculated an activation energy of $5.9 \mathrm{kcal} \mathrm{mol}^{-1}$ that comports well with our experimental value.
For the sake of comparison, we were able to find an alternative iodane structure on the potential energy surface (with a $\mathrm{C}_{\mathrm{ipso}}-\mathrm{I}-\mathrm{F}$ three-centered bond) that represents a plausible pseudorotation intermediate (21-iso);22 it is $25 \mathrm{kcal} \mathrm{mol}^{-1}$ higher in energy than the optimized F-I-F three-centered bond structure (Fig. 4). This number serves as a lower-bound estimate of the barrier to pseudorotation, which would be prohibitively high in energy. Therefore, we can conclude that the observed dynamic activity more likely arises from C-I bond rotation.

\section{Solid-state structure of 21}

In addition to analyzing probe molecule $\mathbf{2 1}$ in solution, we obtained single crystals suitable for X-ray structure determination (Fig. 5). The typical, slightly distorted T-shape arrangement about the iodine atom was observed, with $\theta_{\mathrm{F}-\mathrm{I}-\mathrm{F}}=171.9^{\circ}$, an average $\theta_{\mathrm{C}-\mathrm{I}-\mathrm{F}}=86.0^{\circ}, d(\mathrm{C}-\mathrm{I})=2.097 \AA$, and $d(\mathrm{I}-\mathrm{F})=1.998 \AA$. The $\mathrm{C}_{\text {ortho }}-\mathrm{C}_{\mathrm{ipso}}-\mathrm{I}-\mathrm{F}$ torsion angle $\left(\phi_{\mathrm{C}-\mathrm{C}-\mathrm{I}-\mathrm{F}}\right)$ is $82.9^{\circ}$, placing the $\mathrm{IF}_{2}$ group out of plane with the arene, but not quite perpendicular to it.

Although it is plausible the ortho-substituent influences the orientation of the $\mathrm{IF}_{2}$ group here, it is difficult to separate this
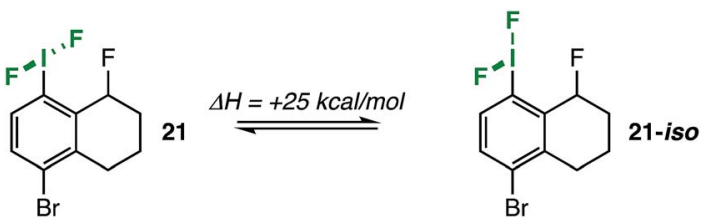

Fig. 4 Isodesmic relationship at wB97xD/cc-pvdz (cc-pvdz-PP for the iodine atom). 


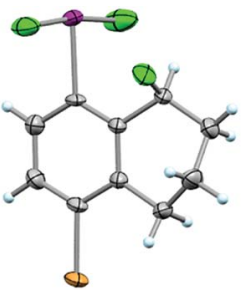

Fig. 5 Crystal structure of compound 21 determined from singlecrystal X-ray diffraction (displacement ellipsoids depicted at $50 \%$ probability level).

phenomenon from packing effects. For instance, only a small number of aryl- $\mathrm{IF}_{2}$ molecular structures have been reported in the CCDC to date, and there are no clear trends yet, for instance, in $\mathrm{C}_{\text {ortho }}-\mathrm{C}_{\mathrm{ipso}}-\mathrm{I}-\mathrm{F}$ torsion angles based on substitution patterns (i.e. presence vs. absence of ortho-substituents, considering ortho-substituents of various sizes, etc.). ${ }^{23}$ Thus, in the solid state, the orientation of the $\mathrm{IF}_{2}$ group is more likely governed by various interactions between the fluorine and iodine atoms of neighboring molecules in the extended lattice than arene substitution pattern (e.g. intermolecular $\mathrm{C}-\mathrm{I} \cdots \mathrm{F}-\mathrm{I}$ contacts of $2.888 \AA$ and I-F $\cdots$ F-I contacts of $2.891 \AA$ were observed in the packing motif of our probe molecule - note $\Sigma r_{\mathrm{V}}[\mathrm{I}, \mathrm{F}]=3.45 \AA$ and $\Sigma r_{\mathrm{V}}[\mathrm{F}, \mathrm{F}]=2.94 \AA$ - and others have reported similar short contacts in aryl-IF $\mathrm{IF}_{2}$ structures). It is also worth mentioning that no short intermolecular contacts involving the Br substituent in $\mathbf{2 1}$ were observed.

\section{An unexpected role of the ortho- substituent}

Although it is clear that the ortho-substituent has an influence on the $\mathrm{IF}_{2}$ group in solution (and potentially in the solid state) from the experiments outlined above, it is not completely understood whether the ortho-substituent actually influences the hydrolytic stability of aryl- $\mathrm{IF}_{2}$ compounds. Thus, we performed a series of controlled hydrolysis experiments, whereby the NMR samples were prepared first under Ar and rigorously dry conditions, then flooded with water immediately prior to the measurements in an attempt to simulate pseudo-first order decays of the aryl- $\mathrm{IF}_{2}$ compounds. $^{24}$

Initially, we varied systematically the size and electronegativity of the ortho-substituents, performing the hydrolysis experiments on compounds 3, 13-15, and $\mathbf{1 7}$ and 18. However, the precipitation of the byproducts seriously complicates the picture (even at lower concentrations), so it was not possible to obtain reliable, quantitative kinetic information or to establish definitive trends. Yet, qualitatively, we can at least note that the ortho-methyl-substituted compound $\mathbf{1 8}$ appears to decay at a similar rate to either the ortho-chloro- or ortho-bromosubstituted compounds 14 and 15 (see ESI $\dagger$ for details). This is an indication that, with respect to hydrolytic stability, the size of the ortho-substituent could play a more important role than electronegativity.
In addition, we varied the position of the trifluoromethyl substituent in the $\mathrm{CF}_{3}$-substituted aryl- $\mathrm{IF}_{2}$ compounds, i.e. investigating compounds $2, \mathbf{2 2}$, and 23. If our hypothesis that the presence of an ortho-substituent has a strong positive effect on hydrolytic stability is correct, then one would expect that compound $\mathbf{2}$ would decay at the slowest rate. Surprisingly, this is not the case. It appears the relative rates of decay based on substitution are: meta $-\mathrm{CF}_{3}>$ ortho $-\mathrm{CF}_{3}>$ para $-\mathrm{CF}_{3}$ (with the para$\mathrm{CF}_{3}$ isomer being the most stable of the three). Based on a thermodynamic argument alone, one would expect the ortho$\mathrm{CF}_{3}$ isomer to decay the fastest, but this is also not the case. Thus, the ortho-substituent may, in fact, have a positive kinetic influence on hydrolytic stability, but it is not nearly as substantial as we anticipated at the outset of this study.

However, by analyzing the reaction mixtures under rigorously air- and moisture-free conditions during the hydrolysis experiments (prior to water addition), we unveiled an interesting and unexpected result when investigating the meta- and para-substituted substrates: formation of tetrafluoro(aryl)- $\lambda^{5}$ iodanes (aryl- $\mathrm{IF}_{4}$ compounds). Aryl-IF $\mathrm{IF}_{4}$ compounds are remarkably more sensitive toward hydrolysis than aryl- $\mathrm{IF}_{2}$ compounds, so extremely careful preparation of the NMR samples was necessary in order to observe them (admittedly, that is how we had completely overlooked this finding earlier in this study). Thus, our initial assumption that the identity of the precipitate forming in the NMR samples prepared in air was strictly the iodosoarene (from hydrolysis of aryl- $\mathrm{IF}_{2}$ ) was incorrect; the precipitate is actually made up of a more significant amount of iodylarene formation for samples employing metaand para-substituted substrates.

This key observation puts the role of the ortho-substituent into a new perspective. The hydrolysis experiments suggest that the ortho-substituent only has a minor positive influence on hydrolytic stability of aryl- $\mathrm{IF}_{2}$ products once they are formed, but it plays a more noteworthy role during the reaction with TCICA and $K F$ in inhibition of further oxidation of iodine(III) to iodine(v).

\section{Substrate scope of tetrafluoro(aryl) $-\lambda^{5}-$ iodanes}

We discovered quickly that satisfactory yields of meta- and parasubstituted aryl- $\mathrm{IF}_{4}$ compounds can be obtained under nearly identical reaction conditions to those in Table 2 (i.e. 4.0 equiv. TCICA and 6.0 equiv. $\mathrm{KF}$ in $\mathrm{MeCN}$ at $40^{\circ} \mathrm{C}$ ) by simply letting the reactions stir for $48 \mathrm{~h}$ instead of $24 \mathrm{~h}$.

Under these conditions, we synthesized a variety of aryl-IF 4 compounds in good yields (79-94\%, Table 4). As anticipated, the functional group compatibility is similar to what we noted in Table 2: aryl- $\mathrm{IF}_{4}$ formation from the unsubstituted iodobenzene (compound 25) and iodoarenes substituted with either electron-withdrawing groups (e.g. halogens, ketones, trifluoromethyl, and pentafluorosulfanyl groups in compounds 26-32) or mild electron-donating groups (e.g. a methyl group in compound 33) proceeds smoothly. On the other hand, the 
Table 4 Substrate scope of meta- and para-substituted aryl-IF 4 compounds $^{a}$

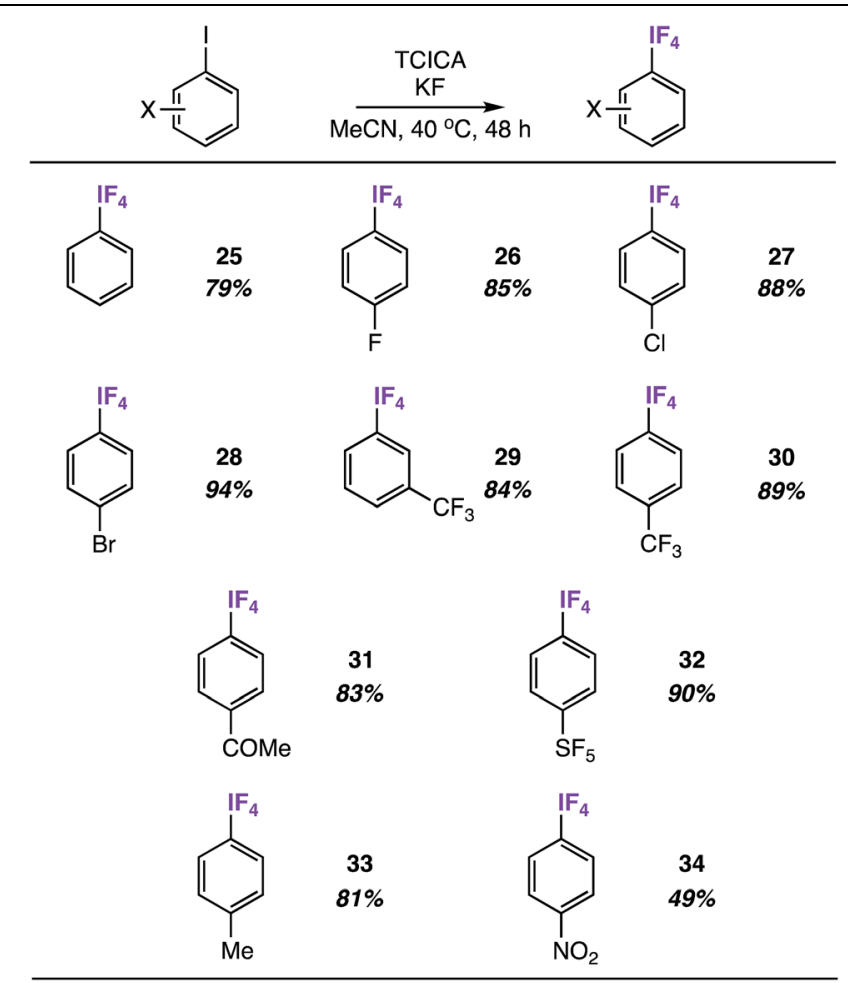

${ }^{a}$ Yields determined by ${ }^{19} \mathrm{~F}$ NMR using fluorobenzene or benzotrifluoride as an internal standard.

substrate with the most extreme electron-withdrawing group a para-nitro group - only provided compound 34 in $49 \%$.

Remarkably, only one ortho-fluorine substituent is enough to stifle $\operatorname{aryl}-\mathrm{IF}_{4}$ formation. For instance, letting 2,4-difluoroiodobenzene stir for $48 \mathrm{~h}$ under reaction conditions results in less than $10 \%$ of the corresponding aryl- $\mathrm{IF}_{4}$ product by ${ }^{19} \mathrm{~F} \mathrm{NMR}$ (note the $\mathrm{IF}_{4}$ signal was identified at $-21.21 \mathrm{ppm}$ in the ${ }^{19} \mathrm{~F}\left\{{ }^{1} \mathrm{H}\right\}$ spectrum as a doublet with a $J$-coupling of $19.2 \mathrm{~Hz}$ to the orthofluorine atom). In nearly all other instances when the orthosubstituent is larger than a fluorine atom, the aryl- $\mathrm{IF}_{2}$ product is formed exclusively.

We also discovered that these aryl- $\mathrm{IF}_{4}$ compounds, although less hydrolytically stable than aryl- $\mathrm{IF}_{2}$ compounds, may be more easily extracted from the reaction mixture by comparison (see $\mathrm{ESI} \dagger$ for details). For instance, we extracted compound $\mathbf{2 6}$ from the reaction mixture under $\mathrm{N}_{2}$ atmosphere in a glovebox using $n$-hexane and obtained a white solid in $77 \%$ isolated yield.

From this, single crystals were grown that proved suitable for Xray structure determination (Fig. 6). As anticipated, a slightly distorted square pyramidal geometry was observed about the iodine atom with the arene in the apical position. In all, the structure displays similar features to the aryl- $\mathrm{IF}_{4}$ compounds originally reported by $\operatorname{Frohn}^{25}$ and $\operatorname{Seppelt}^{26}($ e.g. $d(\mathrm{C}-\mathrm{I})=2.078 \AA$, average $d(\mathrm{I}-$ $\mathrm{F})=1.939 \AA$, average trans $\theta_{\mathrm{F}-\mathrm{I}-\mathrm{F}}=170.1^{\circ}$, average cis $\theta_{\mathrm{F}-\mathrm{I}-\mathrm{F}}=89.6^{\circ}$, average $\theta_{\mathrm{C}-\mathrm{I}-\mathrm{F}}=85.1^{\circ}, \phi_{\mathrm{C}-\mathrm{C}-\mathrm{I}-\mathrm{F}}=44.7^{\circ}$, and short intermolecular $\mathrm{I} \cdots \mathrm{F}$ and $\mathrm{F} \cdots \mathrm{F}$ contacts can be observed in the packing motif).

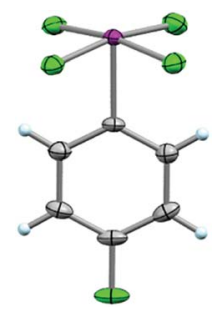

Fig. 6 Crystal structure of compound 26 determined from singlecrystal X-ray diffraction (displacement ellipsoids depicted at 50\% probability level).

\section{Synthesis of another aryl- $\lambda^{5}$-iodane: an ortho-substituted exception}

Inspired by the known reactivity of TCICA and KF in the synthesis of fluoroiodane 36 from alcohol 35 (Fig. 7), ${ }^{27}$ we also wondered if $\mathbf{3 6}$ could be further oxidized to I(v) compound 37 under our reaction conditions. Compound $\mathbf{3 7}$ was originally synthesized by Amey and Martin from 35 using excess $\mathrm{CF}_{3} \mathrm{OF}$, and it was briefly explored as an oxidant. ${ }^{28}$ More recently, it was synthesized in our laboratory using either $\mathrm{XeF}_{2}$ or $\mathrm{Cl}_{2} / \mathrm{KF}^{29}{ }^{29}$ The TCICA/KF approach would make 37 significantly more accessible for future studies.

To our satisfaction, fluoroiodane 36 undergoes further oxidative fluorination under TCICA/KF conditions to provide 37 in $87 \%$ yield by ${ }^{19} \mathrm{~F}$ NMR (Fig. 7). Moreover, under identical reaction conditions, alcohol 35 can also be converted into 37 directly in $69 \%$ yield. In all, our synthesis of 37 indicates that the TCICA/KF approach can be useful in the synthesis of fluorinated $\mathrm{I}(\mathrm{v})$-compounds beyond $\operatorname{aryl}-\mathrm{IF}_{4}$, and it presents a notable "exception to the rule" that ortho-substituted iodoarenes show higher selectivity for the I(III) products over the I(v) products under these reaction conditions.

\section{Solid-state structure of 37}

Additionally, single crystals of 37 suitable for X-ray structure determination were analyzed that display some interesting features (Fig. 8) (note that there were two symmetry-
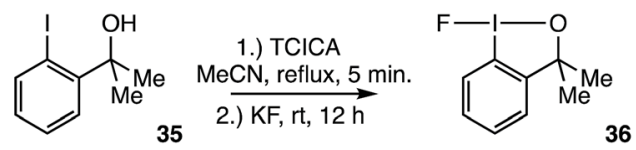

known reactivity

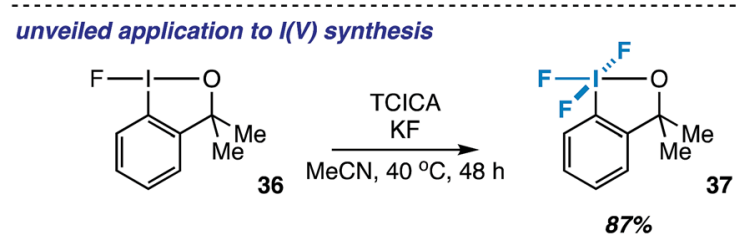

Fig. 7 Extended application to the synthesis of an ortho-substituted I(v) compound (37) as an exception to the rule. Yield determined by ${ }^{19} \mathrm{~F}$ NMR. 


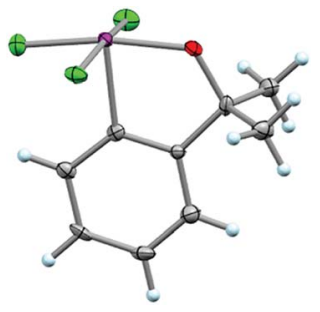

Fig. 8 Crystal structure of compound 37 determined from singlecrystal X-ray diffraction (displacement ellipsoids depicted at $50 \%$ probability level, only one of two asymmetric moieties from asymmetric unit shown).

independent moieties in the asymmetric unit). Due to the participation of the oxygen atom on the ortho-substituent in a three-centered bonding interaction, the average $\mathrm{C}_{\text {ortho }}-\mathrm{C}_{\mathrm{ipso}}{ }^{-}$ I- $\mathrm{F}_{\text {trans }}$ torsion angle $\left(\phi_{\mathrm{C}-\mathrm{C}-\mathrm{I}-\mathrm{F}_{\mathrm{t}}}\right)$ is $171.9^{\circ}$ and the average $\mathrm{C}_{\text {ortho }^{-}}$ $\mathrm{C}_{\mathrm{ipso}}-\mathrm{I}-\mathrm{F}_{\mathrm{cis}}$ torsion angle $\left(\phi_{\mathrm{C}-\mathrm{C}-\mathrm{I}-\mathrm{F}_{\mathrm{o}}}\right)$ is $87.4^{\circ}$. This is a noteworthy deviation from the preferred orientation of the $\mathrm{IF}_{4}$ moiety in aryl-IF ${ }_{4}$ compounds such as 26 , whereby $\phi_{\text {C-C-I-F }} \approx 45^{\circ}$.

Also, the average C-I bond distance $(2.072 \AA)$ is in close accord with aryl- $\mathrm{IF}_{4}$ compound 26 , but the I-F bond distances in 37 are slightly longer, i.e. average $d\left(\mathrm{I}-\mathrm{F}_{\text {cis }}\right)=1.963 \AA$ and average $d\left(\mathrm{I}-\mathrm{F}_{\text {trans }}\right)=1.979 \AA$ (the latter bond conceivably elongated due to the greater trans effect of the oxygen atom; note that the average $d(\mathrm{O}-\mathrm{I})=1.924 \AA) .{ }^{30}$ However, for another comparison, both the $\mathrm{I}-\mathrm{F}_{\text {trans }}$ and $\mathrm{O}-\mathrm{I}$ bonds in $\mathbf{3 7}$ are significantly shorter than those in the fluoroiodane precursor 36 (reported as $2.073 \AA$ and $2.033 \AA$, respectively). ${ }^{29}$

We also noted that the bond angles across the three-centered bonds, on average (trans $\theta_{\mathrm{F}-\mathrm{I}-\mathrm{F}}=168.5^{\circ}$ and trans $\theta_{\mathrm{O}-\mathrm{I}-\mathrm{F}}=$ $167.9^{\circ}$ ), deviate from linearity to a greater extent than aryl-IF compound 26 (trans $\left.\theta_{\mathrm{F}-\mathrm{I}-\mathrm{F}}=170.1^{\circ}\right)$, but slightly less than that of fluoroiodane 36 (trans $\theta_{\mathrm{O}-\mathrm{I}-\mathrm{F}}=167.9^{\circ}$ ) (additional details about bond angle comparisons can be found in the ESI†). Lastly, it is important to mention that the crystal structure of $\mathbf{3 7}$ displayed short intermolecular $\mathrm{I} \cdots \mathrm{F}$ and $\mathrm{F} \cdots \mathrm{F}$ contacts in the packing motif and the tendency to form similar "chain" structures as Frohn, ${ }^{25}$ Seppelt, ${ }^{26}$ and we have observed (see ESI†).

\section{Conclusions}

Remarkably, this TCICA/KF approach to oxidative fluorination of iodoarenes can be employed to synthesize both aryl- $-\mathrm{IF}_{2}$ and aryl- $-\mathrm{IF}_{4}$ compounds. Under our reaction conditions, access to either the I(III)- or I(v)-derivatives is controlled primarily by the substitution pattern on the iodoarene substrate, i.e. an orthosubstituent inhibits further conversion of aryl- $\mathrm{IF}_{2}$ to the corresponding aryl- $\mathrm{IF}_{4}$ compound. An exception to this trend was also presented in the form of compound 37 , whereby the orthosubstituent participates in a three-centered bonding interaction with the iodine atom, thus making the I(v)-derivative accessible. Moreover, this method arguably presents the mildest synthesis of aryl- $\mathrm{IF}_{4}$ compounds reported to date, as well as the mildest approach to electron-deficient aryl- $\mathrm{IF}_{2}$ compounds, as a complement to the methods of Shreeve and Gilmour that can be used to access more electron-rich aryl- $\mathrm{IF}_{2}$ compounds using Selectfluor.

Beyond the development of the TCICA/KF approach as an oxidative fluorination method, this study also raised several questions about the relationship between the $\mathrm{IF}_{2}$ group and ortho-substituents on an arene. This resulted in a series of controlled hydrolysis experiments, computational studies, X-ray crystallographic analyses, and the synthesis of a probe molecule that allotted the first detailed dynamic NMR study on rotation about the C-I bond on substituted aryl- $\mathrm{IF}_{2}$ compounds, from which, rotational barriers and thermodynamic parameters were reported herein.

In all, we hope that our easy access to and increased structural understanding of aryl- $\mathrm{IF}_{2}$ and aryl- $\mathrm{IF}_{4}$ compounds in both solution and the solid state will stimulate more research in this area. Future studies will be focused on studying the oxidative fluorination mechanism, as well as exploring applications of aryl- $\mathrm{IF}_{2}$ and aryl- $\mathrm{IF}_{4}$ compounds as possible reagents.

\section{Conflicts of interest}

There are no conflicts to declare.

\section{Acknowledgements}

We thank the ETH transfer office for support in filing a patent application on this work, in which C. R. P., N. S., and A. T. are listed as inventors. MoBiAS (ETH) is acknowledged for assistance with HRMS analyses. Financial support was provided by ETH Zürich and the ETH Postdoctoral Fellowship Program (C. R. P.).

\section{Notes and references}

1 V. V. Zhdankin, Hypervalent Iodine Chemistry: Preparation, Structure and Synthetic Applications of Polyvalent Iodine Compounds, John Wiley \& Sons, West Sussex, UK, 2014.

2 C. Ye, B. Twamley and J. M. Shreeve, Org. Lett., 2005, 7, 3961.

3 J. C. Sarie, C. Thiehoff, R. J. Mudd, C. G. Daniliuc, G. Kehr and R. Gilmour, J. Org. Chem., 2017, 82, 11792.

4 For reviews, see: (a) P. J. Stang and V. V. Zhdankin, Chem. Rev., 1996, 96, 1123; (b) V. V. Zhdankin, ARKIVOC, 2009, 1; (c) A. Yoshimura and V. V. Zhdankin, Chem. Rev., 2016, 116, 3328.

5 For some examples, see: (a) M. Zupan and A. Pollak, J. Fluorine Chem., 1976, 7, 445; (b) I. Ruppert, J. Fluorine Chem., 1980, 15, 173; (c) D. Naumann and G. Rüther, J. Fluorine Chem., 1980, 15, 213; (d) K. Alam and A. F. Janzen, J. Fluorine Chem., 1987, 36, 179; (e) S. Hara, M. Yoshida, T. Fukuhara and N. Yoneda, Chem. Commun., 1998, 965; $(f)$ V. Padelidakis, W. Tyrra and D. Naumann, J. Fluorine Chem., 1999, 99, 9; $(g)$ H. J. Frohn and V. V. Bardin, J. Fluorine Chem., 2005, 126, 1036; (h) C. Ye, B. Twamley and J. M. Shreeve, Org. Lett., 2005, 7, 3961; (i) S. Suzuki, T. Kamo, K. Fukushi, T. Hiramatsu, E. Tokunaga, T. Dohi, Y. Kita and N. Shibata, Chem. Sci., 2014, 5, 2754; (j) S. M. Banik, J. W. Medley and E. N. Jacobsen, J. Am. Chem. 
Soc., 2016, 138, 5000; (k) J. D. Haupt, M. Berger and S. R. Waldvogel, Org. Lett., 2019, 21, 242.

6 (a) L. M. Yagupol'skii, V. V. Lyalin, V. V. Orda and L. A. Alekseeva, Zh. Obshch. Khim., 1968, 38, 2813; (b) I. I. Maletina, V. V. Orda, N. N. Aleinikov, B. L. Korsunskii and L. M. Yagupol'skii, Zh. Org. Khim., 1976, 12, 1371; (c) H. J. Frohn, Chem.-Ztg., 1984, 108, 146.

7 C. R. Pitts, D. Bornemann, P. Liebing, N. Santschi and A. Togni, Angew. Chem., Int. Ed., 2019, 58, 1950.

8 S. Haubenreisser, T. H. Wöste, C. Martínez, K. Ishihara and K. Muñiz, Angew. Chem., Int. Ed., 2016, 55, 413.

9 U. Tilstam and H. Weinmann, Org. Process Res. Dev., 2002, 6, 384.

10 Gaussian 09, Revision D.01, Gaussian, Inc., Wallingford, CT, 2013.

11 J.-D. Chai and M. Head-Gordon, Phys. Chem. Chem. Phys., 2008, 10, 6615.

12 T. H. Dunning Jr, J. Chem. Phys., 1989, 90, 1007.

13 T. Umemoto, R. P. Singh, Y. Xu and N. Saito, J. Am. Chem. Soc., 2010, 132, 18199.

14 (a) A. L. Johnsen, J. Org. Chem., 1982, 47, 5220; (b) J. R. Wolstenhulme, J. Rosenqvist, O. Lozano, J. Ilupeju, N. Wurz, K. M. Engle, G. W. Pidgeon, P. R. Moore, G. Sandford and V. Gouverneur, Angew. Chem., Int. Ed., 2013, 52, 9796.

15 M. Wang, Q. Fan and X. Jiang, Org. Lett., 2018, 20, 216.

16 The synthesis of one of the tetralone intermediates required to make $\mathbf{2 1}$ is loosely based on a synthesis reported by Kutateladze and co-workers: W. C. Cronk, O. A. Mukhina and A. G. Kutateladze, J. Org. Chem., 2014, 79, 1235. Additional modifications were adapted from procedures in ref. 14, as well as: P. Nguyen, E. Corpuz, T. M. Heidelbaugh, K. Chow and M. E. Garst, J. Org. Chem., 2003, 68, 10195.

17 Note that a temperature calibration was performed prior to the following experiments using $4 \% \mathrm{MeOH}$ in $\mathrm{MeOH}-\mathrm{d} 4$ and a sample of pure $\mathrm{MeOH}-\mathrm{d} 4$, see: A. L. Van Geet, Anal. Chem., 1970, 42, 679.
18 For an example of temperature-dependent shifts in ${ }^{19} \mathrm{~F}$ NMR spectra, see: A. Dimitrov, U. Groß, S. Rüdiger, W. Storek and J. Burdon, J. Fluorine Chem., 1996, 78, 1.

19 (a) T. Drakenberg, K. Dahlqvist and S. Forsén, Acta Chem. Scand., 1970, 24, 694; (b) F. P. Gasparro and N. H. Kolodny, J. Chem. Educ., 1977, 54, 259; (c) F. A. Bovey, Nuclear Magnetic Resonance Spectroscopy, Academic Press, New York, 1988.

20 M. Ochiai, T. Yoshikazu and Y. Masaki, J. Am. Chem. Soc., 1990, 112, 5677.

21 H. J. Reich and C. S. Cooperman, J. Am. Chem. Soc., 1973, 95, 5077.

22 For a review on pseudorotation, see: H. L. Strauss, Annu. Rev. Phys. Chem., 1983, 34, 301.

23 C. R. Groom, I. J. Bruno, M. P. Lightfoot and S. C. Ward, Acta Crystallogr., Sect. B: Struct. Sci., Cryst. Eng. Mater., 2016, 72, 171. The CCDC search was conducted on May 3rd, 2019 using the online version, whereby a similarity search was performed on the core $\mathrm{Ph}-\mathrm{IF}_{2}$ structure. A total of 17 results were found (excluding our contributions), of which, only 8 represented aryl- $\mathrm{IF}_{2}$ compounds (CCDC deposition numbers: 273254, 273255, 1555065, 139385, 938893, 760658, 760659, and 760660) and only 3 represented aryl$\mathrm{IF}_{4}$ compounds (CCDC deposition numbers: 216480, 1409048, and 1308449).

24 J. F. Corbett, J. Chem. Educ., 1972, 49, 663, and references cited therein.

25 H. J. Frohn, S. Görg, G. Henkel and M. Läge, Z. Anorg. Allg. Chem., 1995, 621, 1251.

26 S. Hoyer and K. Seppelt, J. Fluorine Chem., 2004, 125, 989.

27 (a) V. Matoušek, E. Pietrasiak, R. Schwenk and A. Togni, J. Org. Chem., 2013, 78, 6763; (b) J. Charpentier, N. Früh and A. Togni, Chem. Rev., 2015, 115, 650.

28 R. L. Amey and J. C. Martin, J. Am. Chem. Soc., 1979, 101, 5294.

29 J. Charpentier, PhD thesis no. 23352, ETH Zürich, 2016.

30 (a) J. V. Quagliano and L. Schubert, Chem. Rev., 1952, 50, 201; (b) M. Ochiai, T. Sueda, K. Miyamoto, P. Kiprof and V. V. Zhdankin, Angew. Chem., Int. Ed., 2006, 118, 8383. 